\title{
Cerebrovascular Disease and its Relationship to Other Vascular Beds: A Comprehensive Review of the Literature
}

\author{
Julia Slark* and Pankaj Sharma
}

Imperial College Cerebrovascular Research Unit (ICCRU), Hammersmith Hospitals, Imperial College London, Fulham Palace Rd, London W6 8RF, UK

\begin{abstract}
Atherothrombosis, ischaemic heart disease (IHD), cerebrovascular disease (CVD) and peripheral arterial disease (PAD) are a major cause of mortality and are predicted to be the leading cause of death world-wide by 2020 .

It is now recognised that CVD is part of a family of atherothrombotic diseases such as PAD and IHD. However, despite receiving contemporary evidence-based preventative drug therapy, patients with established arterial disease and those with multiple risk factors for atherothrombosis both experience high cardiovascular (CV) event rates with a $4.7 \%$ yearly rate of hard events. People with symptomatic atherosclerosis in 1 vascular bed are at a higher risk of subsequent events in other beds, e.g. patients who suffer a stroke are at a high risk of going on to suffer a coronary event, with a 10 year CV event risk of $42.8 \%$. These findings support the need for increased awareness among physicians and patients for the amount of vascular cross-risk that is related to the overlap between the various beds of atherothrombosis.

Atherothrombosis in stroke patients should be considered as a global arterial disease. We present a systematic review of the literature relating to the risk of non-cerebral (especially sub-clinical) atherothrombotic events in ischaemic stroke patients.
\end{abstract}

Keywords: Atherothrombosis, cardiovascular disease, peripheral artery disease, stroke, secondary prevention.

\section{SCALE OF THE PROBLEM}

Atherothrombosis, ischaemic heart disease (IHD), CVD and PAD are all associated with the main cause of mortality on a world-wide scale and are predicted to be the leading cause of death world-wide by 2020 [1]. In the Health Survey for England [2] in 2003 the prevalence of IHD (angina and heart attack) was $6.4 \%$ in men and $4.1 \%$ in women and with stroke added, the cardiovascular (CV) disease prevalence was $7.9 \%$ and $5.8 \%$, respectively.

Of the 110,000 new stroke occurrences this year in the UK, a third of the patients will go on to have a further stroke. Recurrent stroke is more likely to be fatal than first strokes and survivors are more likely to be left with major disability [3]. However, the circulatory system is not organ-specific. Diseases affecting 1 vascular bed may therefore predict clinical syndromes in other organs. We review the data on likelihood of other organs being affected following a cerebrovascular event.

\section{CROSS-VASCULAR RISK}

It is now recognised that CVD is part of a family of atherothrombotic diseases such as PAD and IHD. Indeed, patients who survive a stroke or Transient Ischaemic Attack

*Address correspondence to this author at the Imperial College Cerebrovascular Research Unit (ICCRU), Hammersmith Hospitals, Imperial College London, Fulham Palace Rd, London W6 8RF, UK;

E-mail: J.slark@imperial.ac.uk
(TIA) are at a relatively high risk for subsequent $\mathrm{CV}$ events, including recurrent stroke, myocardial infarction (MI) and death from vascular causes $[4,5]$ reflecting the shared common risk factors and pathological mechanisms [6].

The CAPRIE [7] (Clopidogrel vs. Aspirin in patients at risk of Ischaemic events) study showed that approximately one quarter of the medium risk individuals had multivascular disease and in 1 in 30 individuals CVD, IHD and PAD were all present. People with symptomatic atherosclerosis in 1 vascular bed are at a higher risk of subsequent events in other beds [8]. Therefore, patients who suffer a stroke are at a high risk of going on to suffer another stroke or heart attack, with a 10 year risk of $42.8 \%$ [9]. These reports have been supported by the findings from the REACH (REduction of Atherothrombosis for Continued Health) registry which evaluated the long term risk of atherothrombotic events in an at risk population. REACH has already demonstrated that a significant proportion of people with PAD, CVD or IHD will have disease in more than 1 vascular location. In 1 year follow-up $8.29 \%$ of patients with CVD went on to have a further vascular event while CV event rates increased significantly with the number of symptomatic locations ( $p=0.0043$ ) [10]. $40 \%$ of patients with CVD demonstrated multi-vascular involvement with many of these affected beds being clinically silent yet ultimately exposing patients to increasing risk profiles with each bed involved.

Steg et al. [11] performed a large international study of stable or high at-risk populations with established atherosclerosis receiving contemporary risk reduction therapies. The 1 
year event rates were high and accrued almost linearly over time and the hard event rates for CV death, MI or stroke increased markedly within the number of symptomatic arterial disease locations. Despite receiving evidence-based preventative drug therapy, this vascular stable population experienced a $4.7 \%$ yearly rate of hard events [11]. During the 1 year follow-up approximately 1 -in-7 patients with established arterial disease experienced either a hard CV event or required hospitalisation for an atherothrombotic event. $\mathrm{Pa}-$ tients with established arterial disease experienced 2-3 times higher event rates than patients with multiple risk factors only.

These data support the need for increased awareness among physicians and patients of the amount of cross-risk that is related to the overlap between the various locations of atherosclerosis.

\section{HEART DISEASE}

There is a risk of 'silent' and asymptomatic IHD in the presence of TIA and stroke [6]. Rokey et al. [12] and Di Pasquale et al. [13] both showed abnormal myocardial imaging and asymptomatic IHD in $41 \%$ and $28 \%$ of their study populations, respectively. Subtypes of ischaemic stroke related to underlying atherosclerosis such as carotid, vertebral or intracranial stenosis, are associated with a higher risk of IHD than non-atherosclerotic subtypes of stroke $[6,14]$.

A systematic review and meta-analysis of the risk of MI and vascular death after TIA and ischaemic stroke showed that after a stroke or TIA the risk of MI and non-stroke vascular death are each $2 \%$ per annum [4]. Other smaller studies suggested that between $25-60 \%$ of stroke patients without any clinical evidence of IHD may have (silent) myocardial ischemia on non invasive tests [6]. While atherosclerosis may explain $20-40 \%$ of stroke, strokes related to atherosclerosis are at a higher risk of MI or non-stroke death than other patients [4].

Several other but smaller studies suggest that patients with carotid stenosis have a high prevalence of IHD $[15,16]$. Sconocchini et al. [16] evaluated 133 patients with asymptomatic and symptomatic significant $(>50 \%)$ carotid stenosis for the presence of IHD using stress-exercise electrocardiography (SE). The study demonstrated that $25 \%$ of patients with no history or IHD had latent myocardial ischemia. They concluded that screening with SE was easy, inexpensive and safe and would allow early detection of those patients who need more active treatments.

Patients with identified carotid, vertebral or intracranial atherosclerosis may therefore be at highest risk of MI or nonstroke death and may be a group which would benefit from $\mathrm{CV}$ investigation on diagnosis of atherosclerosis in a single bed.

\section{RENAL ARTERY DISEASE}

Impaired renal function is a strong independent predictor of CV mortality and morbidity in the absence of any previous history $[17,18]$. Traditional risk factors seem to account for much but not all of this excess risk. There is a significant burden of CVD risk factors among patients with chronic kidney disease (CKD) and these patients are more likely to be treated for hypertension, elevated low density lipoprotein cholesterol (LDL-C) levels, and diabetes, but rates of control of risk factors are uniformly low in those with and without CKD [19]. However, their predisposition to CV disease persists even after adjustment for this overabundance of standard risk factors. Early detection of CKD can not only slow the progression to end-stage renal disease but also identify risk factors for CV disease [20].

\section{Glomerular Filtration Rate (GFR)}

Impaired renal function as measured by low GFR has also been shown to be associated with markers of cerebral small vessel disease on MRI [21]. The population based study of a large sample of elderly people ( $>60$ years) from the Rotterdam Scan Study [22], showed that compared with those with normal kidney performance persons with reduced renal function had a smaller brain volume, smaller deep white matter volume and more white matter lesions and that these findings were independent of CV risk factors. GFR may be an easy and readily measureable indicator of cerebral small vessel disease. Understanding the interplay of vascular disease between the kidney and the brain may hold the promise of identifying novel strategies to reduce the risk of damage to either organ [23].

\section{Cystatin C}

Cystatin $\mathrm{C}$ is a serum protein that is filtered out of the blood by the kidneys and serves as a measure of kidney function. Cystatin $\mathrm{C}$ is produced steadily by all types of nucleated cells in the body. Deo et al. [24] performed a study for the Health, Ageing and Body composition study, to evaluate whether impaired kidney function predicted the risk of fatal $\mathrm{CV}$ disease independent of prevalent and incident $\mathrm{CV}$ events. Higher Cystatin-C concentrations were significantly associated with CV disease [24].

\section{Serum Creatinine}

Although end-stage renal disease substantially increases the risks of death and CV disease [25], the effects of less severe kidney dysfunction on these outcomes are less well defined [26, 27]. The HOPE study aimed to determine whether mild renal insufficiency increased $\mathrm{CV}$ risk and whether ramipril, an angiotensin converting enzyme inhibitor, decreased that risk. 980 patients with mild renal insufficiency measured by an increased serum creatinine $(>1.4$ $\mathrm{mg} / \mathrm{dL} ; 124 \mu \mathrm{mol} /$ ) and 8307 patients with normal renal function were investigated for a primary outcome measure of $\mathrm{CV}$ death, MI or stroke. The cumulative incidence of the primary outcome was higher in patients with renal insufficiency than those without. Ramipril reduced the incidence of the primary outcome in patients with and without renal insufficiency. The study showed that patients with pre-existing vascular disease or diabetes combined with an additional CV risk factor and mild renal insufficiency had a significantly increased risk for subsequent $\mathrm{CV}$ events [27] but importantly, this risk was reversible.

Shlipak et al. [28] concluded through a study aimed to determine the prevalence of elevated risk through serum creatinine levels $(>1.4 \mathrm{mg} / \mathrm{dL} ; 124 \mu \mathrm{mol}$ in women and $>1.5$ 
$\mathrm{mg} / \mathrm{dL} ; 133 \mu \mathrm{mol} / \mathrm{L}$ in men) that renal insufficiency is a marker for elevated CVD risk in elderly ( $>65$ years age) adults. Older individuals demonstrated more significant differences in hypertension prevalence, treatment and control. The prevalence of hypertension was significantly higher among those with compared with those without CKD. These differences were more evident among older individuals, consistent with previously published data demonstrating lower rates of blood pressure (BP) control among older individuals with mild to moderate CKD [19]. Poor kidney function is also highly prevalent in the elderly population together with more widespread small vessel disease in the brain [21].

Whether CV disease is a risk factor for the progression or development of worsening kidney disease was studied by Elsayed et al. [17]. This study looked at 13,826 individuals, used an increase in serum creatinine level and a reduced estimated GFR (eGFR) as markers of a kidney function decline. They concluded that $\mathrm{CV}$ disease is indeed independently associated with kidney function decline and the development of CKD. This is an important finding which reiterates the importance of the need to be aware of the interconnectors between diseases and the independent impact one has on the other. PAD has also been shown to have a high prevalence in patients with renal insufficiency [29].

Both the kidney and brain are low resistance end-organs and are exposed to high-volume blood flow [30]. The similarities in the vascular supply to these organs could be useful as vascular disease in 1 organ may inform us about vascular disease in the other [23]. Prevention and treatment of CVD are major considerations in the management of individuals with CKD. Less is known regarding the CVD risk factor burden among individuals with earlier stages of CKD, many of whom are not necessarily treated for CKD or do not manifest clinical CVD. However, relatively minor renal abnormalities such as a slightly reduced GFR or microalbuminuria, but even within normal ranges, may be associated with increased risk of vascular events [30]. Moderate CKD is an independent risk factor for $\mathrm{CV}$ disease, suggesting a need to better understand $\mathrm{CV}$ risk factor burden and rates of risk factor treatment and control in this group.

The limitation with evaluation studies of the prevalence of renal disease in association with CV disease is that different studies appear to use different markers for diagnosis of renal disease, with some using GFR, some cystatin $\mathrm{C}$ and others serum creatinine levels. However, all these measures are well defined biochemical markers of reduced kidney function but nevertheless this possibly blurs the association between renal disease and $\mathrm{CV}$ disease risk, as each marker used for renal disease may have an alternative independent influence on atherosclerotic events.

\section{PERIPHERAL ARTERIAL DISEASE (PAD)}

PAD is defined by atherosclerotic obstruction of the abdominal aorta and arteries to the legs that reduces arterial flow during exercise and/or at rest [31]. Approximately one third of patients with PAD have typical claudication defined as pain in 1 or both legs on walking, primarily affecting the calves, that does not go away with continued walking and is relieved by rest. In patients with claudication, the severity of the condition increases slowly; $25 \%$ have worsening claudi- cation, and 5\% undergo an amputation within 5 years. Less than $5-10 \%$ of patients have critical leg ischaemia (ischaemic pain in the distal foot, ischemic ulceration or gangrene), but their risk of limb loss is substantial [32]. Only $\sim 10-30 \%$ of patients with PAD present with classic symptoms of intermittent claudication [31]. This may be a reason for its under-diagnosis and clinicians reliant on a classic history of claudication could miss the majority of cases [33].

This is of concern as patients with PAD, even in the absence of a history of MI or ischaemic stroke, have approximately the same relative risk of death from $\mathrm{CV}$ causes as patients with a history of IHD or CVD [32]. The ageadjusted prevalence of PAD is approximately $12 \%$, and the disorder affects men and women equally. Patients with PAD are usually regarded as a group that is at particularly high risk of cardiac ischaemic events, yet PAD is commonly both under-diagnosed and undertreated [11].

Vickrey et al. [34] found that the PAD cohort in their study of secondary ishaemic events among persons with atherosclerotic vascular disease experienced the highest case fatality or proportion of secondary events that were fatal stroke, fatal MI and other vascular deaths. The REACH registry findings support these findings with PAD patients experiencing the highest rates of $\mathrm{CV}$ death and major $\mathrm{CV}$ events due to an atherothrombotic event [11]. However, Janes et al. [35] performed a multivariate analysis that showed compared to patients with IHD, patients with PAD had decreased use of antiplatelet agents, statins and ACE inhibitors and during hospital admission, treatment was significantly less likely to be initiated in patients with PAD.

\section{ANKLE BRACHIAL INDEX (ABI)}

The ABI may be the most reliable detector of PAD [31]. It compares systolic BP in the dorsarlis pedis and/or posterior tibial arteries of the lower limbs to that of the brachial artery with the use of a hand-held Doppler device [31]. As this is the simplest, inexpensive, reliable and reproducible method of identifying PAD it may be used on patients with a history of CV disease and stroke, to easily identify if there is atherosclerosis in another vascular bed resulting in a further increase in the risk of vascular disease. The Scandinavian Simvastatin Survival Study [36] revealed that the use of simvastatin reduced episodes of new or worsening intermittent claudication providing evidence that if treated with $\mathrm{CV}$ disease risk factor management strategies, PAD patients can have a positive outcome. The Heart Protection Study (HPS) [56] went on to conclude that simvastatin not only reduced the risk of a first event being suffered by a person but also reduced the risks of subsequent events for many types of high -risk patients including those with PAD .

\section{RETINAL VESSEL DISEASE}

A study to determine if hypertensive retinopathy (HTR) is an indicator of silent brain infarction (SBI) in asymptomatic hypertensive subjects using MRI and retinal photography has been undertaken [37]. 14\% of subjects had the presence of HTR and, following multivariate analysis, age (OR, 1.07; 95\% CI, 1.03 - 1.10) and HTR (OR, 2.01 for grade 1; OR, 3.03 for grade 2) were the independent indicators for the presence of SBI. The higher the grade of HTR, the more 
prevalent SBI than in persons with normal retina (by linear association test, $\mathrm{p}=0.001$ ).

Mitchell et al. [38] performed a study investigating retinal microvascular signs as a potential risk of stroke and stroke mortality. The purpose of this study was to assess the relation of retinal microvascular signs and incident stroke and stroke mortality in an older population. The authors took retinal photographs on baseline participants (3,654 patients aged 49+ years) of the Blue Mountains Eye Study (1992 to 1994). They assessed the presence of retinopathy (microaneurysms, retinal haemorrhages) in participants without diabetes and retinal arteriolar signs in all participants using standardized grading protocols. Incident stroke/TIA/CVD deaths (combined stroke events) were identified at follow-up examinations during 1997 - 1999. During a 7-year period, 859 participants died, 97 (11.3\%) of which died of CVD causes. Of survivors, 24 had confirmed incident stroke, and 11 had incident TIA. Combined stroke events were more frequent in participants with retinopathy $(5.7 \%)$, with moderate/severe arteriovenous nicking $(4.2 \%)$, or with focal arteriolar narrowing $(7.2 \%)$ compared with those without (1.9\%). After controlling for age, sex, systolic BP, smoking, and self-rated health, retinopathy was significantly associated with combined stroke events (relative risk [RR] 1.7, 95\% CI 1.0 to 2.8 ) in persons without diabetes. This association was stronger in those without severe hypertension (RR 2.7, CI 1.2 to 6.2 ) or in persons with 2 or more retinal microvascular signs (RR 2.7, CI 1.5 to 5.2). Generalized or focal arteriolar narrowing or arteriovenous nicking was not independently associated with combined stroke events after multivariate adjustment. They concluded that in older Australians without diabetes, retinopathy signs predict stroke or stroke-related death independent of traditional stroke risk factors.

Mead et al. [39] performed a comparison study of risk factors in patients with transient and prolonged eye and brain ischemic events. It was the largest study to date to compare risk factors in ischaemic stroke, cerebral TIA, retinal artery occlusion (RAO) and amaurosis fugax. The results showed that severe ipsilateral carotid stenosis was more common in eye than brain events and atrial fibrillation was more common in brain than eye events. Atrial fibrillation was more often associated with prolonged (stroke and RAO) than transient (cerebral TIA and amaurosis fugax) symptoms. These data suggest that brain and eye events reflect distinct patterns of vascular disease and risk factors that probably have relevance to the pathophysiology of the event and its prognosis. They suggest focusing management of subgroups of patients with different cerebrovascular ischemic symptoms to target stroke prevention more effectively.

\section{RETINAL VEIN DISEASE}

Patients with retinal vein occlusions (RVO) are at increased risk of CV disease [40]. The risk of future CV disease was determined by this group using the Framingham algorithm and the risk estimated to guide decision about preventative treatment for $\mathrm{CV}$ disease in this patient group. The study concluded that RVO is a presenting complaint in a group of patients at increased risk of CV disease. They agreed that long-term follow up data demonstrates an increased risk of mortality from $\mathrm{CV}$ disease in patients with
RVO. For patients presenting with RVO, this may be the first indication of developing atherosclerosis and could be used as an early indicator and initiation of treatment may be helpful at this time.

\section{ESTABLISHED RISK FACTORS}

Risk factors for vascular diseases such as diabetes, obesity and hypertension as well as the presence of renal dysfunction lead to activation of the renin-angiotensin system and oxidative stress. This leads to elevated asymmetric dimethylarginine (ADMA), low-grade inflammation and dyslipidemia which are all common pathophysiological mechanisms that play a role in this association with vascular disease [30].

\section{DIABETES}

Diabetes remains an independent risk factor for systemic atherosclerosis and increases the risk of atherogenesis via detrimental effects on the vessel wall [31]. Diabetes mellitus is more associated with small vessel disease than large vessel disease. Surprisingly Touze et al. [4] found no correlation between diabetes mellitus prevalence at baseline and risk of MI during follow-up and suggested the link between diabetes mellitus and small vessel disease as a possible explanation for this. The association between diabetes and small vessel disease does appear to carry a low risk of MI during followup [41].

\section{PRE-HYPERTENSION AND HYPERTENSION}

Individuals with pre-hypertensive levels of $\mathrm{BP}$, as defined by $120 / 80$ to $139 / 89 \mathrm{mmHg}$, have an increased risk of developing $\mathrm{CV}$ disease and stroke compared with those with optimal BP $<120 / 80 \mathrm{mmHg}$. The association is among blacks, those with diabetes mellitus and those with a high BMI [42].

An overview of reviews of the effects on stroke seen in trials of BP-lowering drugs and compared these with the results available from cohort studies has been performed [43]. Cohort studies now indicate that in the Asia Pacific region as well as in North America and Western Europe, each $10 \mathrm{mmHg}$ lower systolic BP is associated with a decrease in risk of stroke of approximately one third in subjects aged 60-79 years. The association is continuous down to levels of at least $115 / 75 \mathrm{mmHg}$ and is consistent across sexes, regions, and stroke subtypes and for fatal and nonfatal events. The proportional association is age dependent but is still strong and positive in those aged 80 years. Data from randomized controlled trials, in which mean age at event was approximately 70 years, indicate that a $10 \mathrm{mmHg}$ reduction in systolic BP is associated with a reduction in risk of stroke of approximately one third. Per $\mathrm{mmHg}$ systolic BP reduction, the relative benefits for stroke appear similar between agents, by baseline BP levels, and whether or not individuals have a past history of CVD. There is, however, evidence of greater benefit with a larger BP reduction. Epidemiologically expected benefits of BP lowering for stroke risk reduction are broadly consistent across a range of different population subgroups with greater benefits from larger BP reductions. 


\section{HYPERLIPIDAEMIA}

Hyperlipidaemia is a well established risk factor for $\mathrm{CV}$ disease [56]. It initiates atherosclerosis by disturbing the normal homeostatic function of the endothelium and vascular wall [44]. Raised LDL levels in the plasma increase the rate of delivery and retention of LDL in the arterial wall, this then leads to reactive oxygen species depleting antioxidants to oxidize fatty acids on the LDL surface transforming it to oxidized LDL particles. Oxidized LDL will activate the overlying endothelial cells, the combination will go on to inhibit the production of nitric oxide and rapidly convert the available nitric oxide to the inactive metabolite. This drop in nitric oxide causes detrimental effects on the vascular wall and in turn causes recruitment of inflammatory cells and leads to atherosclerotic lesions [44]. A large amount of data has been collected to support the use of statin therapy to reduce $\mathrm{CV}$ risk and several recent meta-analyses assessing the risk reduction on statin therapy have shown that statin therapy provides high levels of protection for all-cause mortality and non-haemorrhagic strokes and other vascular diseases such as CKD and CAD [36, 45-47].

\section{METABOLIC SYNDROME}

The Metabolic Syndrome is characterised by a number of vascular risk factors such as, elevated BP, high blood glucose, obesity and hyperlipidaemia. Boden-Albala et al. [48] performed a study looking at the association between the metabolic syndrome and stroke. The study was part of the Northern Manhattan Study and 3298 stroke-free community residents were prospectively followed up for a mean of 6.4 years. The metabolic syndrome was defined according to the National Cholesterol Education Program Adult Treatment Panel III guidelines. Cox proportional-hazards models were used to calculate hazard ratios (HRs) and 95\% CIs for ischemic stroke and vascular events (ischemic stroke, MI, or vascular death). The study showed that more than $44 \%$ of the cohort had the metabolic syndrome (48\% of women $v s .38 \%$ of men, $P$ 0.0001), which was more prevalent among Hispanics $(50 \%)$ than whites $(39 \%)$ or blacks $(37 \%)$. The metabolic syndrome was associated with increased risk of stroke (HR_1.5; 95\% CI, 1.1 to 2.2) and vascular events (HR_1.6; $95 \% \mathrm{CI}, 1.3$ to 2.0 ) after adjustment for socio-demographic and risk factors. The effect of the metabolic syndrome on stroke risk was greater among women (HR_2.0; 95\% CI, 1.3 to 3.1 ) than men (HR_1.1; 95\% CI, 0.6 to 1.9 ) and among Hispanics (HR_2.0; $95 \%$ CI, 1.2 to 3.4) compared with blacks and whites. The etiologic fraction estimates suggest that elimination of the metabolic syndrome would result in a $19 \%$ reduction in overall stroke, a $30 \%$ reduction of stroke in women; and a $35 \%$ reduction of stroke among Hispanics.

However, there is still debate about the metabolic syndrome as an independent risk factor for CVD with the absolute $\mathrm{CV}$ risk of the metabolic syndrome not necessarily higher than those of its individual components [49]. The prevalence of the metabolic syndrome varies according to definition, ethnicity and gender[49].

Subjects with the metabolic syndrome are at increased CV risk but whether it is a useful CV risk marker above and beyond the risk associated with its individual components remains uncertain.

\section{RISK ASSESSMENT}

The Framingham study has been used for 30 years to produce 'health risk appraisal' systems to predict primary and subsequent risk of IHD and stroke. The risk appraisal models have proven to be useful to clinicians in understanding the multifactorial nature of IHD in particular [50] but is limited by being based mainly on the predominantly 'white' population. Notwithstanding this limitation, the length of the study provides us with the most comprehensive overview of the effects of CV disease available to date.

D'Agostino et al. [50] and Wolfe et al. [51] used the Framingham study risk models to identify cross-risk for IHD, stroke and diabetes. They found the models to be useful tools to gain a better understanding of the relationship between risk factors and the occurrences of CVD events in subjects who are free of CVD, as well as individuals who have had a prior event or are at particularly high risk of an event [50].

The Joint British Society guidelines suggest that all adults from 40 years onwards, who have no history of CVD or diabetes, and who are not already on treatment for BP or lipids, should be considered for an opportunistic comprehensive CVD risk assessment in primary care [52]. Younger adults ( $<40$ years) with a family history of premature atherosclerotic disease should also have their CV risk factors measured. Risk assessment should include ethnicity, smoking habit history, family history of CVD, and measurements of weight, waist circumference, BP, non-fasting lipids (total cholesterol and HDL cholesterol), and non-fasting glucose. Those not found at this comprehensive CV risk assessment to be at high total CVD risk based on the Joint British Societies' charts [52], or started for other reasons on drug therapy to lower BP, lipids, or glucose, should have their risk assessment repeated, ideally within 5 years. Under the age of 40 years the 10 year total CVD risk will usually be low but the risk in the coming years, assuming risk factors do not change, can be tracked forward to older age groups. Over the age of 70 years CVD risk is usually $>20 \%$ over 10 years, especially for men, but they suggest that total CVD risk should still be formally estimated. However, this will underestimate the true total CVD risk of a person older than 70 years. For people with established atherosclerotic CV disease, hypertension with target organ damage, familial dyslipidaemias such as familial hypercholesterolaemia, or diabetes, formal risk estimation is not necessary as all these people are at high total CVD risk and their risk factors should be treated.

\section{MANAGING MULTIPLE RISK FACTORS IN THE MULTIVASCULAR PATIENT}

Comprehensive approaches to the control of CV diseases have resulted in a $60 \%$ decline in mortality rates over a 25 year period [53]. At least four-fifths of recurrent vascular events in patients with $\mathrm{CV}$ diseases might be prevented by the application of a comprehensive, multi-factorial approach [5]. Following a comprehensive literature review [5] to identify meta-analyses of randomized controlled trials reporting on the efficacy of secondary prevention strategies, strategies were combined on a multiplicative scale and cumulative risk reduction were computed over a 5 year interval. The combi- 
nation of 5 proven strategies applied to survivors of an initial stroke or TIA including, dietary modification, exercise, aspirin, statin and antihypertensive agent could result in an $80 \%$ risk reduction with a number needed to treat of $\sim 5$. However, compliance with treatment is usually substantially lower in routine clinical practice than in closely monitored and motivated trial populations and it is still up to the healthcare professional to educate the patient in a way that helps them to understand the level of risk and the need for lifestyle and medical modifications [3].

Van Wijk et al. [54] looked at the long-term determinants of survival and risk of vascular events following TIA or minor ischaemic stroke and found that approximately 10 years after a first event $54 \%$ of the participants had experienced at least 1 new vascular event. They found that the risk of a vascular event was highest shortly after the ischaemic event and reached its lowest point at about 3 years and then gradually rose again afterwards. One explanation they gave for the gradual rise of increased risk after the 3 year period was the possible decline in drug compliance and from the reduced attention to lifestyle factors in follow-up. In light of the substantial risk of recurrence of subsequent ischaemic events and advances in therapeutic treatments which have demonstrated to be effective for secondary prevention, Vickrey et al. [34] suggest that further research should be performed to look at assessment and care patterns for secondary prevention to determine whether there are gaps between actual care and the evidence based recommendations. This could be useful in light of the study by Qureshi et al. [55], who reported in 1252 survivors of MI and stroke from the National Health and Nutrition Examination Survey 3, that only 35\% of hypertensive's were controlled. Blood glucose in diabetes was controlled in only about $50 \%$ and cholesterol was poorly controlled in $46 \%$. About $18 \%$ of individuals were also still smoking cigarettes.

\section{CONCLUSIONS}

Cerebrovascular disease is associated with vascular disease occurring elsewhere in other vascular beds. This should not surprise us as the arterial tree is not restricted to specific organs and an event in 1 target region is merely the clinical manifestation of the total burden of atherosclerosis in the entire vascular tree. The association of asymptomatic and symptomatic multiple locations of atherothrombosis with high event rates suggest that atherosclerosis should be addressed as a global arterial disease.

\section{ACKNOWLEDGEMENTS}

PS holds a DoH Senior Fellowship.

JS is funded by a Hammersmith Hospitals Special Trustees award.

Research in ICCRU is funded by grants from the DoH, Heart Research UK, Stroke Association, Hammersmith Hospital Trustees, Wellcome Trust, Henry Smith Charity and pharmaceutical companies.

\section{CONFLICT OF INTEREST}

PS has received honoraria for lecturing in industrysponsored meetings and has received industry funding for attending national and international meetings. PS has received research grants from pharmaceutical companies and has been a paid consultant to the biotech industry and is/has been a member of industry advisory boards.

JS has no conflict of interest.

\begin{tabular}{|c|c|c|}
\hline \multicolumn{3}{|c|}{ ABBREVIATIONS } \\
\hline $\mathrm{ABI}$ & $=$ & Ankle Brachial Index \\
\hline BMI & $=$ & Body Mass Index \\
\hline $\mathrm{BP}$ & $=$ & Blood Pressure \\
\hline CHD & $=$ & Coronary Heart Disease \\
\hline CKD & $=$ & Chronic Kidney Disease \\
\hline $\mathrm{CV}$ & $=$ & Cardiovascular \\
\hline CVD & $=$ & Cerebrovascular Disease \\
\hline eGFR & $=$ & Estimated Glomerular Filtration Rate \\
\hline GFR & $=$ & Glomerular Filtration Rate \\
\hline HTR & $=$ & Hypertensive Retinopathy \\
\hline IHD & $=$ & Ischaemic Heart Disease \\
\hline LDL-C & $=$ & Low Density Lipoprotein - Cholesterol \\
\hline MI & $=$ & Myocardial Infarction \\
\hline PAD & $=$ & Peripheral Artery Disease \\
\hline $\mathrm{RAO}$ & $=$ & Retinal Artery Occlusion \\
\hline RVO & $=$ & Retinal Vein Occlusions \\
\hline SBI & $=$ & Silent Brain Infarction \\
\hline SE & $=$ & stress-exercise electrocardiography \\
\hline TIA & $=$ & Transient Ischaemic Attack \\
\hline
\end{tabular}

\section{REFERENCES}

[1] Bhatt D, Steg PG. Reaching for new heights in disease management. Br J Cardiol 2007; 14: 190.

[2] Department of Health. Health survey for England (2003). http://www.dh.gov.uk/PublicationsAndStatistics/PublishedSurvey/ HealthSurveyForEngland/fs/en

[3] Rothwell P. Making the most of secondary prevention. Stroke 2007; 38: 1726 .

[4] Touze E, Varenne O, Chatellier G, et al. Risk of Myocardial infarction and vascular death after TIA and Ischaemic stroke. A systematic review and meta-analysis. Stroke 2005; 36: 2748-55.

[5] Hackam D, Spence D. Combining multiple approaches for the secondary prevention of vascular events after stroke. Stroke 2007; 38: $1881-5$.

[6] Adams R, Chimowitz M, Alpert J, et al. Coronary risk evaluation in patients with TIA and Stroke. A Scientific statement for healthcare professionals from the stroke council and the council on clinical cardiology of the American Heart Association/American Stroke Association. Circulation 2003; 108: 1278-90

[7] CAPRIE Steering Committee. A randomised, blinded, trial of Clopidogrel vs. aspirin in patients at risk of ischaemic events (CAPRIE). Lancet 1996; 348: 1329-39.

[8] Barnett A, Begg A, Bradbury A, et al. Panel of the Atherothrombosis clinical faculty group. Clopidogrel in the modern management of atherothrombosis. Br J Cardiol 2007; 14 (Suppl 5): S3-S10. 
[9] Jenkinson D. Implications of REACH for stroke physicians. Br J Cardiol 2007; 14 (Suppl 3): S11-S12.

[10] Morrell JM, Kassianos GC. One-year data from the UK arm of the REACH Registry. Br J Cardiol 2007; 14: 215-8.

[11] Steg PG, Bhatt DL, Wilson PWF, et al. One year cardiovascular event rates in outpatients with atherothrombosis. JAMA 2007; 297: 1197-206.

[12] Rokey R, Harati Y, Rolak LA. Coronary artery disease in patients with cerebrovascular disease: a prospective study. Ann Neurol 1984; 16: 50-53.

[13] Di Pasquale G, Andreoli A, Pinelli G. Cerebral Ischaemia and asymptomatic coronary artery disease: a prospective study of 83 patients. Stroke 1986; 17: 1098-101.

[14] Rothwell P, Coull AJ, Silver LE, et al.; for the Oxford Vascular Study. Population-based study of event-rate, incidence, case fatality and mortality for all acute vascular events in all arterial territories. Lancet 2005; 366: 1773-83.

[15] Urbinati S, Di Pasquale G, Andreoli A. Frequency and prognostic significance of silent coronary artery disease in patients with cerebral ischaemia undergoing carotid endarterectomy. Am J Cardiol 1992; 69:1166-70.

[16] Sconocchini C, Racco F, Pratillo G. Patients with carotid stenosis and clinical history negative for coronary disease: usefulness of the ergometric test for the identification of ischemic myocardial disease. Minerva Med 1997; 88: 173-81.

[17] Elsayed E, Tighiouart H, Griffith J, et al. Cardiovascular Disease and subsequent kidney disease. Arch Intern Med 2007; 167: 11306.

[18] Townsend RR. Stroke in chronic kidney disease: prevention and management. Clin J Am Soc Nephrol 2008; (3 Suppl 1): S11-6.

[19] Parikh N, Hwang S-J, Larson M, et al. Cardiovascular disease risk factors in chronic kidney disease: overall burden and rate of treatment and control. Arch Intern Med 2006; 166: 1884-91.

[20] Hostetter T. Chronic kidney disease predicts cardiovascular disease. N Engl J Med 2004; 351: 1344-6.

[21] Ikram A, Vernooij MW, Hofman A, et al. Kidney function is related to cerebral small vessel disease. Stroke 2008; 39: 55-61.

[22] Vermeer SE, Koudstaal PJ, Oudkerk M, et al. Retinal vessel diameters and cerebral small vessel disease: the Rotterdam Scan Study. Brain 2006; 129: 182-8.

[23] Seliger SL, Longstreth WT Jr. Lessons about brain vascular disease from another pulsating organ, the kidney. Stroke 2008; 39: 5-6.

[24] Deo R, Wassel-Fyr C, Fried L, et al. Kidney dysfunction and fatal cardiovascular disease: an association independent of atherosclerotic events: Results from the Health, Ageing and body composition study. Am Heart J 2008; 155: 62-8.

[25] Seliger SL, Gillen DL, Longstreth WT Jr, et al. Elevated risk of stroke among patients with end-stage renal disease. Kidney Int 2003; 64: 603-9.

[26] Go AS, Chertow GM, Fan D, et al. Chronic kidney disease and the risks of death, cardiovascular events, and hospitalization. N Engl J Med 2004; 351: 1296-305.

[27] Mann JFE, Gerstein HC, Pogue J, et al., for the HOPE investigators. Renal insufficiency as a predictor of cardiovascular outcomes and the impact of Ramipril: The HOPE randomized trial. Ann Int Med 2001; 134: 629-36.

[28] Shlipak MG, Heidenreich PA, Noguchi H, et al. Association of renal insufficiency with treatment and outcomes after Myocardial infarction in elderly patients. Ann Int Med 2002; 137: 555-62.

[29] O'Hare AM, Glidden DV, Fox CS, et al. High prevalence of peripheral arterial disease in person with renal insufficiency: results from the National Health and Nutrition Examination survey 19992000. Circulation 2004; 109: 320-3.

[30] Schiffrin EL, Lipman ML, Mann JF. Chronic kidney disease: effects on the cardiovascular system. Circulation 2007; 116: 85-97

[31] Chi Y-W, Jaff MR. Optimal risk factor modification and medical management of the patient with peripheral arterial disease. Catheteriz Cardiovasc Intervent 2008; 71: 475-89.

[32] Hiatt WR. Medical treatment in peripheral arterial disease and claudication. N Engl J Med 2001; 344: 1608-21.
[33] Hirsch AT, Criqui MH, Treat-Jacobson D, et al. Peripheral arterial disease detection, awareness and treatment in primary care. JAMA 2001; 286: 1317-24.

[34] Vickrey B, Rector T, Wickstrom S, et al. Occurrence of secondary ischaemic events among person with atherosclerotic vascular disease. Stroke 2002; 33: 901-6.

[35] Janes S, West J, Hopkinson B, et al. Pharmacological secondary prevention in people with peripheral arterial disease compared to those with coronary artery disease: a missed opportunity. $\mathrm{Br} \mathrm{J}$ Cardiol 2008; 15: 48-50

[36] Pedersen T, Kjekshus J, Pyorala K, et al. Effect of Simvastatin on ischaemic signs and symptoms in the Scandinavian Simvastatin survival study. Am J Cardiol 1998; 81: 333-5.

[37] Kwon HM, Kim BJ, Oh JY, et al. Retinopathy as an indicator of silent brain infarction in asymptomatic hypertensive subjects. J Neurol Sci 2007; 252; 159-62.

[38] Mitchell P, Wang JJ, Wong T, et al. Retinal microvascular signs and risk of stroke and stroke mortality. Neurology 2005; 65: 10059.

[39] Mead G, Lewis S, Wardlaw JM, et al. Comparison of risk factors in patients with transient and prolonged eye and brain ischaemic syndromes. Stroke 2002; 33: 2383-90.

[40] Martin SC, Butcher A, Martin N, et al. Cardiovascular risk assessment in patients with retinal vein occlusion. Br J Ophthal 2002; 86: 774-6.

[41] Diener HC, Bogousslavsky J, Brass LM, et al. Aspirin and Clopidogrel compared with Clopidogrel alone after recent ischaemic stroke or TIA in high risk patients. (MATCH): randomised, double blind, placebo-controlled trial. Lancet 2004; 364: 331-7.

[42] Kshirsagar AV, Carpenter M, Bang H, et al. Blood pressure usually considered normal is associated with an elevated risk of cardiovascular disease. Am J Med 2007; 119: 133-41.

[43] Lawes CM, Bennett DA, Feigin VL, et al. Blood pressure and stroke: an overview of published reviews. Stroke 2004; 35: 2237-8.

[44] Kinlay S, Libby P, Ganz P. Endothelial function and coronary artery disease. Curr Opin Lipidol 2001; 12: 383-9.

[45] O'Regan C, Wu P, Arora P, et al. Statin therapy in stroke prevention: meta-analysis involving 121,000 patients. Am J Med 2008; 121: 24-33.

[46] Strippoli GF, Navaneethan SD, Johnson DW, et al. Effects of statins in patients with chronic kidney disease: meta-analysis and meta regression of randomised controlled trials. BMJ 2008; 336: 624-5.

[47] Gould AL, Davies GM, Alemao E, et al. Cholesterol reduction yields clinical benefits: meta analysis including recent trials. Clin Ther 2007; 29: 778-94.

[48] Boden-Albala B, Sacco RL, Lee H-S, et al. Metabolic syndrome and ischaemic stroke: Northern Manhattan Study. Stroke 2008; 39: 30-5.

[49] Qiao Q, Gao W, Zhang L, et al. Metabolic syndrome and cardiovascular disease. Ann Clin Biochem 2007; 44: 232-63.

[50] D'Agostino RB, Russell MW, Huse Dm, et al. Primary and subsequent coronary risk appraisal: New results from the Framingham study. Am Heart J 2000; 139: 272-81.

[51] Wolfe PA, D'Agostino RB, Belange AJ, et al. Probability of stroke: a risk profile from the Framingham Study. Stroke 1991; 22: 312-8.

[52] Joint British Societies' guidelines on prevention of cardiovascular disease in clinical practice. Prepared by British Cardiac society, British Hypertension society, Diabetes UK, HEART UK, Primary Care Cardiovascular society, The Stroke Association. Heart 2005; 91: 1-52.

[53] World Health Organisation. The World Health Report 2002: Reducing Risks, Promoting Healthy Life. Geneva, Switzerland: World Health Organisation 2002.

[54] Van Wijk I, Kappelle LJ, Van Gijn J, et al. for the LiLAC study. Long-term survival and vascular event risk after transient ischaemic attach o0r minor ischaemic stroke: a cohort study. Lancet 2005; 365: 2098-104.

[55] Qureshi A, Suri MFK, Guterman LR, et al. Ineffective secondary prevention in survivors of cardiovascular events in the U.S population: report from the third national health and nutrition examination survey. Arch Inter Med 2001; 161: 1621-8. 
[56] Heart Protection Study Collaborative Group. MRC/BHF Heart Protection Study of Cholesterol lowering with Simvastatin in
20536 high risk individuals: a randomised placebo-controlled trial. Lancet 2002; 360: 7-22.

Received: June 03, 2008

Revised: July 04,2008

Accepted: July 04, 2008

(C) Slark and Sharma; Licensee Bentham Open.

This is an open access article licensed under the terms of the Creative Commons Attribution Non-Commercial License

(http://creativecommons.org/licenses/by-nc/3.0/) which permits unrestricted, non-commercial use, distribution and reproduction in any medium, provided the work is properly cited. 